PROCEEDINGS OF THE

AMERICAN MATHEMATICAL SOCIETY

Volume 140, Number 7, July 2012, Pages 2357-2373

S 0002-9939(2011)11071-2

Article electronically published on October 26, 2011

\title{
STRUCTURE AND BIFURCATION OF PULLBACK ATTRACTORS IN A NON-AUTONOMOUS CHAFEE-INFANTE EQUATION
}

\author{
A. N. CARVALHO, J. A. LANGA, AND J. C. ROBINSON
}

(Communicated by Yingfei Yi)

\begin{abstract}
The Chafee-Infante equation is one of the canonical infinite-dimensional dynamical systems for which a complete description of the global attractor is available. In this paper we study the structure of the pullback attractor for a non-autonomous version of this equation, $u_{t}=u_{x x}+\lambda u-\beta(t) u^{3}$, and investigate the bifurcations that this attractor undergoes as $\lambda$ is varied. We are able to describe these in some detail, despite the fact that our model is truly non-autonomous; i.e., we do not restrict to 'small perturbations' of the autonomous case.
\end{abstract}

\section{INTRODUCTION}

The study of the internal dynamics on the global attractor is a very important subject in the theory of infinite-dimensional dynamical systems. This problem is usually closely related to the geometric structure of the attractor and its bifurcations under perturbation. However, a detailed description of the global attractor of an infinite-dimensional dynamical system is usually a difficult task, and there is only a small set of examples for which a full characterization of the attractor is available. One of these canonical models is the Chafee-Infante equation, for which the attractor consists of an odd number of stationary points (which bifurcate from the origin) and the unstable manifolds joining them (Hale [7]; Henry [8], ChafeeInfante [5]; Robinson [17]).

In this paper we will study the asymptotic behaviour of the following nonautonomous version of the Chafee-Infante equation:

$$
\left\{\begin{array}{l}
u_{t}=u_{x x}+\lambda u-\beta(t) u^{3}, 0 \leq x \leq \pi \text { and } t>\tau, \\
u(0, t)=u(\pi, t)=0 \\
u(x, \tau)=\phi(x)
\end{array}\right.
$$

Received by the editors September 14, 2010 and, in revised form, February 15, 2011.

2010 Mathematics Subject Classification. Primary 35B32, 35B40, 35B41, 37L30.

The first author was partially supported by CNPq 302022/2008-2, CAPES/DGU 267/2008 and FAPESP 2008/55516-3, Brazil.

The second author was partially supported by Ministerio de Ciencia e Innovación grants \#MTM2008-0088, \#PBH2006-0003-PC, and Junta de Andalucía grants \#P07-FQM-02468, \#FQM314 and \#HF2008-0039, Spain.

The third author is currently an EPSRC Leadership Fellow, grant \#EP/G007470/1. 
where $\lambda \in[0, \infty)$ and $\phi \in X:=H_{0}^{1}(0, \pi)$. Throughout we denote by $\lambda_{n}$ the eigenvalues of the operator $u \mapsto-u_{x x}$ with Dirichlet boundary conditions on $(0, \pi)$ (so that in fact $\lambda_{n}=n^{2}$ ). Note also that there are a variety of natural phase spaces in which one can consider the dynamics of this equation; in addition to $H_{0}^{1}(0, \pi)$, one might consider $L^{2}(0, \pi)$ or $C^{0}([0, \pi])$. For consistency we choose the smallest space $H_{0}^{1}(0, \pi)$; we could work in larger spaces of initial conditions, but since the equation is immediately smoothing, little is lost by proceeding in this way.

Carvalho and Langa 2, 4, and their collaborators [3] have studied the characterization of pullback attractors related to small non-autonomous perturbations of autonomous dynamical systems possessing a global attractor. As a consequence of these results, for (1.1) it is already known that if $\beta(t)$ is a small non-autonomous perturbation of an autonomous $\beta_{0}$, then the associated pullback attractor can be described in a similar manner as the global attractor for the autonomous case (see Section (4). One can consider asymptotically autonomous systems as a special case of this result. It is also known that the pullback attractors behave upper and lower semicontinuously with respect to the limiting (autonomous) global attractor [12, [2].

In this paper we want to study the asymptotic dynamics of (1.1) when $\beta(t)$ is 'genuinely' non-autonomous, i.e. when we do not have a small non-autonomous perturbation of an underlying autonomous system. The most general case we consider is when there are positive constants $\beta_{1}$ and $\beta_{2}$ such that

$$
0<\beta_{1} \leq \beta(t) \leq \beta_{2} \quad \text { for all } t \in \mathbb{R} .
$$

First, we show in Section 3 that much of the structure of the autonomous attractor is preserved; its dimension has the same dependence on $\lambda$ (independent of the choice of $\beta(t)$ ), and for any chosen $\lambda>0$ the attractor has 'at least as much complexity' as the autonomous attractor (as reflected by the number of 'equilibria', if defined appropriately). However, we are not able to go as far as a full description of the structure of the attractor.

In Section 4 we restrict to small non-autonomous perturbations. In this case, for each fixed $\lambda>0$ we can give a complete description of the pullback attractor, provided that the perturbation is sufficiently small. We then show that for any $\Lambda>0$ this is enough to give a 'rough' bifurcation diagram for all $\lambda \leq \Lambda$, provided we ignore the (admittedly most interesting) behaviour around the autonomous bifurcation values $\lambda=\lambda_{n}$.

In the final section we consider some other situations in which we can characterize the pullback attractor, but in which $\beta(t)$ is not a small perturbation of an autonomous system (i.e. is 'truly non-autonomous'): in each of these situations there is a change of variables that transforms the original non-autonomous system into a small perturbation of some autonomous equation, and we can use similar ideas to those in Section 4 to describe the attractors.

While some of the results here are consequences of our previous work (in particular in the papers [2], 3], and 4] cited above), our approach in what follows is to bring to bear all the tools currently at our disposal to give as detailed a description as we can of the non-autonomous Chafee-Infante equation, and to promote it as a canonical model of non-autonomous dynamics. Indeed, since the autonomous version is the equation in which our knowledge of the structure of the attractor is the deepest, it is to be expected that the pursuit of a similar goal in the non-autonomous 
case will serve as an important test case in which to develop the correct language in which to consider the dynamics of more general non-autonomous systems.

\section{The autonomous Chafee-Infante equation}

One of the few models in which we can fully understand the structure of the global attractor is the autonomous Chafee-Infante reaction-diffusion equation:

$$
\left\{\begin{array}{l}
u_{t}=u_{x x}+\lambda u-u^{3}, 0 \leq x \leq \pi \text { and } t>0, \\
u(0, t)=u(\pi, t)=0, \\
u(x, 0)=\phi(x),
\end{array}\right.
$$

where $\lambda \in[0, \infty)$ and $\phi \in X:=H_{0}^{1}(0, \pi)$. For this autonomous problem we follow [5], 7] and [8]. Existence and uniqueness for this equation can be found in many references (see, for example, 8). Simple energy estimates show that these solutions do not blow up in $X$ and, for each $t \geq 0$, we can define the operator $S(t): X \rightarrow X$ via

$$
S(t) \phi=u(t ; \phi)
$$

where $u(t, \phi) \in X$ denotes the unique solution of (2.1). The family of operators $\{S(t): t \geq 0\}$ satisfies the usual semigroup properties, namely $S(0)=\mathrm{Id}$, $S(t) S(s)=S(t+s)$ for all $t, s \geq 0$, and $(t, \phi) \mapsto S(t) \phi$ is continuous as a map from $\mathbb{R} \times X$ into $X$.

It can also be shown that this semigroup $\{S(t): t \geq 0\}$ possesses a global attractor $\mathcal{A}$, a compact invariant set that attracts bounded subsets of $X$ :

$$
S(t) \mathcal{A}=\mathcal{A} \text { for every } t \in \mathbb{R} \text { (invariance) }
$$

and

$$
\operatorname{dist}(S(t) B, \mathcal{A}) \rightarrow 0 \quad \text { as } t \rightarrow \infty \text { (attraction), }
$$

where $B$ is any bounded subset of $X$ and $\operatorname{dist}(A, B)=: \sup _{a \in A} \inf _{b \in B}\|a-b\|_{X}$ is the Hausdorff semidistance between $A$ and $B$.

This attractor has finite upper box-counting dimension (often termed 'fractal dimension' in the literature), which can be estimated from above in terms of $\lambda$ (see [6, Theorem III.5.2),

$$
\operatorname{dim}(\mathcal{A}) \leq \sqrt{3} \lambda^{1 / 2}
$$

(Although this bound is derived for the dimension measured in $L^{2}(0, \pi)$, one can then use the invariance of $\mathcal{A}$ and the fact that $S(1): L^{2}(0,1) \rightarrow H_{0}^{1}(0, \pi)$ is Lipschitz to deduce that the same bound holds if one measures the dimension in $H_{0}^{1}(0, \pi)$, since the box-counting dimension does not increase under Lipschitz maps; cf. Proposition VI.3.2 in [18.)

In order to study the bifurcation of the equilibria for (2.1) we seek the non-trivial solutions of the boundary value problem

$$
u_{x x}-\lambda u+u^{3}=0, \quad u(0)=u(\pi)=0,
$$

for $0 \leq \lambda<\infty$. This problem is well understood: for $\lambda \in\left(n^{2},(n+1)^{2}\right]$ there are $2 n+1$ stationary points $\left\{0, u_{1}^{ \pm}, \ldots, u_{n}^{ \pm}\right\}$, where $u_{j}^{ \pm}(\lambda)$ bifurcate from the origin at $\lambda=\lambda_{j}:=j^{2}$. Each bifurcation is associated with an increase in the dimension of the local unstable manifold near the origin by one; this provides a lower bound on the attractor dimension which is of the same order as the upper bound in (2.4): $\operatorname{dim}(\mathcal{A}) \geq n$ for $\lambda \in\left(\lambda_{n}, \lambda_{n+1}\right]$, and hence $\operatorname{dim}(\mathcal{A})=O\left(\lambda^{1 / 2}\right)$. 
Moreover, the semigroup $S(\cdot)$ associated to (2.1) is a gradient system [7, 8]; that is, there exists an associated Lyapunov functional $V: X \rightarrow \mathbb{R}$. In particular, therefore, $\mathcal{A}$ is a gradient-like attractor (see [3]); i.e. it is given as the union of the unstable manifolds of its equilibria,

$$
\mathcal{A}=\bigcup_{i=0}^{n} W^{\mathrm{u}}\left(u_{i}^{ \pm}\right)
$$

with the convention that $u_{0}^{ \pm}=0$. If $y(\cdot): \mathbb{R} \rightarrow X$ is a global solution for (2.1), there are $i, j \in\{0, \ldots, n\}$ such that

$$
\lim _{t \rightarrow-\infty} y(t)=u_{j}^{*} \quad \text { and } \quad \lim _{t \rightarrow+\infty} y(t)=u_{i}^{*},
$$

where $V\left(u_{i}^{*}\right)<V\left(u_{j}^{*}\right)$.

In particular, as $\lambda$ passes through $\lambda_{1}$, the attractor changes from a single stable fixed point $(u \equiv 0)$ to a set, homeomorphic to an interval, which consists of the one-dimensional unstable manifold of the origin together with $u_{1}^{ \pm}$. This has two distinct components, one of which lies in the cone of positive solutions and on which all solutions approach a new positive fixed point, and one which lies in the cone of negative solutions and on which all solutions approach a new negative fixed point. We will be able to recover elements of this first bifurcation in the next section for a very general non-autonomous form of the equation.

\section{Structure of the Pullback attractor When $\beta_{1} \leq \beta(t) \leq \beta_{2}$}

In this section we treat the case of a general bounded non-autonomous term. We are not able to obtain as much detailed information as we can in more restrictive cases, but nevertheless we are able to show 'lower bounds' on the complexity of the pullback attractor that increase with $\lambda$ in line with the autonomous case.

The dynamics of non-autonomous equations is described by a non-linear process, i.e. a two-parameter family $\{S(t, \tau): t \geq \tau \in \mathbb{R}\}$ of continuous operators from $X$ into itself such that $S(\tau, \tau)=I, S(t, \sigma) S(\sigma, \tau)=S(t, \tau)$, for each $t \geq \sigma \geq \tau$, and $\left(t, \tau, z_{0}\right) \mapsto S(t, \tau) z_{0}$ is continuous for $t \geq \tau, z_{0} \in X$.

Definition 1. A family of compact sets $\{A(t) \subset X: t \in \mathbb{R}\}$ is the pullback attractor for $\{S(t, \tau): t \geq \tau \in \mathbb{R}\}$ if it is invariant $(S(t, s) A(s)=A(t)$, for all $t \geq s)$, where $\bigcup_{s<t} A(s)$ is bounded for each $t \in \mathbb{R}$ and attracts all bounded subsets of $X$ 'in the pullback sense'; that is, for each bounded subset $B$ of $X$,

$$
\lim _{\tau \rightarrow-\infty} \operatorname{dist}(S(t, \tau) B, A(t))=0, \forall t \in \mathbb{R} .
$$

(Note that the requirement in the definition that $\bigcup_{s \leq t} A(s)$ is bounded for each $t \in \mathbb{R}$ is not strictly necessary; or, rather, one can choose to allow sets for which this is not the case as 'pullback attractors'. However, requiring $A(\cdot)$ to be 'bounded in the past' in this way excludes certainly pathologies, e.g. unstable sets that are not contained in the attractor; see [10] for more details.)

3.1. Asymptotic bounds on solutions. In this section we give some global bounds for the pullback attractor for a general bounded $\beta(t)$ and show that this attractor consists of all the globally defined solutions. We assume that there are positive constants $\beta_{1}, \beta_{2}$ such that $0<\beta_{1} \leq \beta(t) \leq \beta_{2}$ for all $t \in \mathbb{R}$ and consider the non-autonomous equation

$$
u_{t}=u_{x x}+\lambda u-\beta(t) u^{3}
$$


for $x \in[0, \pi]$ with Dirichlet boundary conditions. The existence of global pullback attractors for this equation is known (see, for instance, [14). Moreover, the results in [11, 13] provide the following upper and lower bounds for bounded global solutions to (3.1). In particular, the pullback attractor lies between two 'extremal bounded solutions'. [In the following result the choice $X=C^{0}([0,1])$ would in fact be sufficient.]

Theorem 2. There exist two extremal (minimal and maximal) bounded global solutions, $\xi_{m}(\cdot)$ and $\xi_{M}(\cdot)$ for (3.1), i.e. if $\psi(\cdot)$ is any bounded global solution for $S(t, s)$, then

$$
\xi_{m}(t) \leq \psi(t) \leq \xi_{M}(t), \text { for all } t \in \mathbb{R}
$$

Moreover, the order interval $I(t)=\left[\xi_{m}(t), \xi_{M}(t)\right]=\left\{z \in X: \xi_{m}(t) \leq z \leq \xi_{M}(t)\right\}$ is forwards invariant, and the extremal solutions are pullback bounds for solutions of (3.1) in the sense that

$$
\xi_{m}(t) \leq \liminf _{s \rightarrow-\infty} u\left(t, s, x ; u_{0}\right) \leq \limsup _{s \rightarrow-\infty} u\left(t, s, x ; u_{0}\right) \leq \xi_{M}(t)
$$

uniformly in $u_{0} \in D \subset X$ bounded.

Moreover, if $u_{0} \in X$ and $u_{0} \geq \xi_{M}(s)$ for all $s \in \mathbb{R}$, then

$$
\lim _{s \rightarrow-\infty} u\left(t, s, x ; u_{0}\right)=\xi_{M}(t)
$$

i.e. $\xi_{M}(\cdot)$ is pullback asymptotically stable from above. Similarly, $\xi_{m}(\cdot)$ is pullback asymptotically stable from below; i.e. if $u_{0} \in X$ and $u_{0} \leq \xi_{m}(s)$ for all $s \in \mathbb{R}$, then

$$
\lim _{s \rightarrow-\infty} u\left(t, s, x ; u_{0}\right)=\xi_{m}(t)
$$

As a consequence, (3.1) has a pullback attractor $A(t)$ with

$$
A(t) \subset\left[\xi_{m}(t), \xi_{M}(t)\right], \text { for all } t \in \mathbb{R},
$$

and with $\xi_{m}(t), \xi_{M}(t) \in A(t)$ for all $t \in \mathbb{R}$.

When the pullback attractor is uniformly bounded in $X$ (uniformly with respect to $t$ ) the definition we have given of the pullback attractor has a similar characterization to that possible in the autonomous case (see [3], []. $)$, namely as the union of all the globally defined bounded orbits:

$$
\{A(t): t \in \mathbb{R}\}=\{\xi(t): \xi(\cdot): \mathbb{R} \rightarrow \mathcal{Z} \text { is bounded and } S(t, \tau) \xi(\tau)=\xi(t)\} .
$$

In principle, there could be unbounded global solutions which lie outside the attractor. This is a common feature of non-autonomous dynamical systems (cf. [10]), but in our case every global solution of (3.1) is uniformly bounded in $X$ (with respect to $t \in \mathbb{R})$ as a consequence of the strong dissipation caused by the term $-\beta(t) u^{3}$ (see Remark III.1.3 in [18]).

3.2. Upper and lower bounds on the dimension of the pullback attractor. Before analysing further the structure of the pullback attractor in the general case of a bounded $\beta(t)$, we first consider bounds on its box-counting dimension.

Observe that Theorem 2 gives upper and lower bounds for solutions in the pullback attractor $A(t)$. Moreover, the upper and lower bounds on $\beta(t)$ imply that

$$
\xi_{M}(t) \leq w_{\beta_{1}} \quad \text { for all } t \in \mathbb{R}
$$


where $w_{\beta_{1}}$ is the unique positive equilibrium of the autonomous Chafee-Infante equation (3.1) with $\beta(t) \equiv \beta_{1}$. Thus, we conclude that

$$
\bigcup_{t \in \mathbb{R}} A(t) \subset B_{X}\left(0,\left\|w_{\beta_{1}}\right\|_{X}\right)
$$

i.e. the pullback attractor is bounded uniformly in $X$ (with respect to $t$ ).

It follows that our equation (3.1) is a particular case of the non-autonomous reaction-diffusion equation considered in Section VIII.4.2 in Chepyzhov and Vishik 6]; their Theorem VIII.4.2 applies, and so

$$
\operatorname{dim}(A(t)) \leq \sqrt{3} \lambda \quad \text { for all } t \in \mathbb{R},
$$

where the dimension is calculated in $L^{2}(0,1)$. In line with the comments above, one can obtain the same bound in $H_{0}^{1}(0,1)$ using the invariance of $A(\cdot)$ and the Lipschitz property of the map $S(t+1, t)$.

On the other hand, $u \equiv 0$ is an equilibrium solution of the non-autonomous equation (3.1). Moreover, it is hyperbolic, since the linearization of (3.1) around zero gives

$$
v_{t}-\Delta v=\left(\lambda-3 \beta(t) u^{2}\right)_{\lfloor u=0} v=\lambda v,
$$

so that, by the results in [8], if $\lambda_{n} \leq \lambda<\lambda_{n+1}$, then the local unstable manifold at zero is an $n$-dimensional Lipschitz manifold. Since this must be part of the pullback attractor, $\operatorname{dim}(A(t))$ is bounded below by $n \simeq \lambda^{1 / 2}$. It follows that in the non-autonomous case, as in the autonomous case, the dimension of the attractor is $O\left(\lambda^{1 / 2}\right)$.

Observe that there is certainly a bifurcation within the pullback attractor (in the sense of an attractor bifurcation as defined by Ma and Wang [15]) as $\lambda$ passes through each successive eigenvalue of the negative Laplacian, since the dimension of the unstable manifold of the origin increases. In the next section we are able to obtain some more information on the change in the structure of the whole pullback attractor.

3.3. On the attractor in the positive cone. Observe that since the non-linear term is odd if $u$ is a solution of (3.1), then so is $v=-u$. As a consequence, the behaviour of solutions in the positive and negative cones (defined formally below) are symmetric, and thus, if we denote by $\xi_{M}(t)$ the maximal bounded solution in the positive cone, the minimal bounded solution in the negative cone is just $-\xi_{M}(t)$.

We consider the positive cone within $H_{0}^{1}(0, \pi)$,

$$
\mathcal{V}^{+}=\left\{u \in H_{0}^{1}(0, \pi): u(x) \geq 0 \text { for a.e. } x \in[0, \pi]\right\} .
$$

For (3.1), we can define an order with respect to $\mathcal{V}^{+}$; i.e., $u_{0} \leq v_{0}$ if $v_{0}-u_{0} \in \mathcal{V}^{+}$.

In order to investigate further the behaviour of positive solutions, the following definition (11]) is crucial.

Definition 3. A positive function with values in $C(\bar{\Omega})$ is non-degenerate at $\infty$ $($ respectively $-\infty)$ if there exists $t_{0} \in \mathbb{R}$ such that $u$ is defined in $\left[t_{0}, \infty\right.$ ) (respectively $\left.\left(-\infty, t_{0}\right]\right)$ and there exists a $C^{1}(\bar{\Omega})$ function $\varphi_{0}(x)>0$ in $\Omega$ (vanishing on $\partial \Omega$ in case of Dirichlet boundary conditions) and satisfying $\frac{\partial \varphi_{0}}{\partial n}<0$, such that

$$
u(t, x) \geq \varphi_{0}(x) \quad \text { for all } x \in \Omega \text { and all } t \geq t_{0}
$$

(respectively for all $t \leq t_{0}$ ). 
As a direct application of the results in [11] we obtain the following description of the pullback attractor within the positive cone.

\section{Theorem 4.}

a) If $\lambda<\lambda_{1}$, then $\xi_{M}(t) \equiv 0$ for all $t \in \mathbb{R}$.

b) If $\lambda>\lambda_{1}$, then $\xi_{M}(t)$ is strictly positive and is the unique non-degenerate global solution at $-\infty$ and $+\infty$.

c) The pullback attractor for (3.1) in the positive cone satisfies $A^{+}(t) \subset$ $\left[0, \xi_{M}(t)\right]$. In particular, any global solution in $A^{+}(t)$ is non-degenerate at $-\infty$. Finally, $\xi_{M}(t)$ also attracts forwards in time all bounded sets lying in the interior of the positive cone.

As a consequence, this result also provides an example of a 'non-autonomous pitchfork bifurcation' in the sense of [10.

Corollary 5. Equation (3.1) undergoes a localised pitchfork bifurcation at $u \equiv 0$, $\lambda=\lambda_{1}$; i.e. there exists a $\delta>0$ such that

(i) for all $\lambda_{1}-\delta<\lambda \leq \lambda_{1}$ the zero solution is pullback attracting;

(ii) when $\lambda_{1}<\lambda<\lambda_{1}+\delta$ the zero solution is asymptotically unstable, and there exist bounded trajectories $\xi_{M}^{+}(t)$ and $\xi_{M}^{-}(t)$ that are pullback (and forwards) attracting in $\mathcal{O}_{\delta}(0) \cap \mathcal{V}^{+}$and $\mathcal{O}_{\delta}(0) \cap \mathcal{V}^{-}$, respectively, and satisfy

$$
\xi_{M}^{ \pm}(t) \rightarrow 0 \text { as } \lambda \searrow \lambda_{1}
$$

uniformly on compact subsets of $\mathbb{R}$.

Currently we are able to give a full description of the pullback attractor only when we restrict our attention to the cone of positive solutions (part (c) of Theorem 4). While a complete description in general currently seems out of reach, one interesting open problem which appears more tractable is to determine whether, for $0<\lambda-\lambda_{1}<\delta$ and $\delta$ small enough, the pullback attractor on all of $X$ is in fact the closure of the one-dimensional unstable manifold of the zero solution (i.e. the union of those in the positive and negative cones).

3.4. Global hyperbolic solutions within the pullback attractor. One way to analyse further the asymptotic behaviour of (3.1),

$$
u_{t}=u_{x x}+\lambda u-\beta(t) u^{3}, \quad u(s)=u_{s},
$$

is to scale the solutions according to

$$
z(x, t)=\beta(t)^{\frac{1}{2}} u(x, t),
$$

so that $z$ satisfies

$$
\left\{\begin{array}{l}
z_{t}=z_{x x}+\left(\lambda+\frac{1}{2} \frac{\beta^{\prime}(t)}{\beta(t)}\right) z-z^{3}, \\
z(0, t)=z(\pi, t)=0 \\
z(s)=z_{s}=\beta(s)^{\frac{1}{2}} u_{s}
\end{array}\right.
$$

the non-autonomous dependence has been transferred to the linear part of the equation.

As indicated in Section 2, if $\lambda \in\left(\lambda_{n}, \lambda_{n+1}\right)$, then for any $\beta>0$, the problem (3.1) with $\beta(t) \equiv \beta$ has $2 n+1$ equilibria, all of them hyperbolic. It is reasonable to expect that even if $\beta(t)$ depends on time there will be the same number of global hyperbolic solutions. The following result goes some way towards establishing this. 
Theorem 6. Consider (3.1) with $0<\beta_{1} \leq \beta(t) \leq \beta_{2}$. Then there exists an $n_{0}$ and a sequence $\mu_{n} \rightarrow \infty$ with $\mu_{n} \in\left(\lambda_{n}, \lambda_{n+1}\right)$ such that, for all $n \geq n_{0}$, corresponding to each of the $2 n+1$ equilibria of

$$
u_{t}=u_{x x}+\mu_{j} u-u^{3}
$$

there is a global hyperbolic solution contained in the pullback attractor of

$$
u_{t}=u_{x x}+\mu_{j} u-\beta(t) u^{3} .
$$

Proof. Choose $\mu_{n}=\lambda_{n}+n$, so that $\lambda_{n+1}-\mu_{n}=n+1$; in particular, $\lambda_{n}<\mu_{n}<$ $\lambda_{n+1}$. Consider the problem

$$
u_{t}=u_{x x}+\mu_{n} u-u^{3}
$$

and denote the equilibria of this equation by $u_{0} \equiv 0$ and $u_{1}^{ \pm}\left(\mu_{n}\right), \ldots, u_{n}^{ \pm}\left(\mu_{n}\right)$. If we linearize the equation around each of these equilibria we obtain

$$
u_{t}=u_{x x}+\mu_{n} u-3\left(u_{i}^{ \pm}\right)^{2} u \text {. }
$$

If we multiply the equation by $\frac{1}{n}$ and rescale time, then

$$
u_{t}=\frac{1}{n} u_{x x}+\frac{\mu_{n}}{n} u-\frac{3}{n}\left(u_{i}^{ \pm}\right)^{2} u .
$$

Noticing that the semigroup generated by $\frac{1}{n} u_{x x}+\frac{\mu_{n}}{n} u$ has an exponential dichotomy with constant 1 and exponent 1 , we see that a perturbation of the type $\frac{1}{2 n} \frac{\beta^{\prime}(t)}{\beta(t)}$ with $n$ suitably large will not destroy the exponential dichotomy (independently of $i$ or \pm ). So, there is an $n_{0} \in \mathbb{N}$ such that, for each $n \geq n_{0}$, the problem (3.1) has at least $2 n+1$ global hyperbolic solutions (see [2]).

3.5. 'Non-autonomous equilibria' within the pullback attractor. We now prove a related and in some ways stronger result, although this involves dropping the requirement of hyperbolicity. First we define the notion of a 'non-autonomous equilibrium' of (3.1) using the lap number $l(u)$, which denotes the number of zeros of $u(x)$.

Recall, from [16, that for a solution of the (autonomous or non-autonomous) Chafee-Infante equation the lap number $l(t):=l(u(t))$ is decreasing in $t$ and, for the autonomous case, the only global solutions for which $l(t)$ is constant for all $t \geq 0$ are the equilibria (in which case, of course, all the zeros are fixed). This gives a characterization of the equilibria of the autonomous problem in terms of the lap-number function $l(t)$.

We use this as the basis of a definition in the non-autonomous case; it would be natural to make the same requirement (that $l(t)$ is constant for all $t \in \mathbb{R}$ ), but since the solutions are non-autonomous the zeros can move and there is the possibility that their number could change 'in the limit' $t= \pm \infty$. We therefore make the following somewhat less elegant definition, which should be viewed as a first attempt towards a more systematic use of the lap number in this non-autonomous context.

Definition 7. We say that a global solution $\xi: \mathbb{R} \rightarrow X$ for (3.1) is a nonautonomous equilibrium if there is a $\delta>0$ such that the distance between two consecutive zeroes of $\xi(t)$ is greater than $\delta$ for all $t \in \mathbb{R}$. (In particular, $l(t)$ is constant for all $t \in \mathbb{R}$.) 
If $\lambda \in\left(\lambda_{N}, \lambda_{N+1}\right)$, then there are $2 N+1$ equilibria $\left\{0, \phi_{1, j}^{ \pm}, \ldots, \phi_{N, j}^{ \pm}\right\}$for both of the two problems

$$
u_{t}=u_{x x}+\lambda u-\beta_{j} u^{3},
$$

$j=1,2$, and that $\phi_{i, 2}^{ \pm}=\beta^{\frac{1}{2}} \phi_{i, 1}^{ \pm}$, where $\beta=\frac{\beta_{1}}{\beta_{2}}$. Thus all the $i+1$ zeros of $\phi_{i, 1}^{ \pm}$and $\phi_{i, 2}^{ \pm}$coincide.

It follows from the dynamical method used to construct the solutions $\phi_{i, 1}^{ \pm}$(see [5, 7. 8, 17]) that the zeros of $\phi_{i, j}^{ \pm}$are $0, \frac{\pi}{i}, \frac{2 \pi}{i}, \ldots, \pi$, for $1 \leq i \leq N$ and $j=$ 1,2. Furthermore, these solutions have certain symmetry properties (which we now introduce) that will play a key role in our proof. Define

$$
Y_{i}^{ \pm}=\left\{\phi \in H_{0}^{1}(0, \pi): \min \left(\phi_{i, 1}^{ \pm}(x), \phi_{i, 2}^{ \pm}(x)\right) \leq \phi(x) \leq \max \left(\phi_{i, 1}^{ \pm}(x), \phi_{i, 2}^{ \pm}(x)\right)\right\}
$$

and $X_{i}^{ \pm}=Y_{i}^{ \pm} \cap Z_{i}^{ \pm}$, where

$$
\begin{array}{r}
Z_{i}^{ \pm}=\left\{\phi \in H_{0}^{1}(0, \pi): \phi(x)=\phi\left(\frac{2 p-1}{i} \pi-x\right) \text { for } x \in\left(0, \frac{2 p-1}{i} \pi\right), 1 \leq p \leq \frac{i+1}{2}\right. \\
\text { and } \left.\phi(x)=-\phi\left(\frac{2 q}{i} \pi-x\right) \text { for } x \in\left(0, \frac{2 q}{i} \pi\right), 1 \leq q \leq \frac{i}{2}\right\}
\end{array}
$$

The solutions $\phi_{i, j}^{ \pm}$are elements of $X_{i}^{ \pm}$; again, this follows from the dynamical construction referred to above.

We now show that each $X_{i}^{ \pm}$is positively invariant under the dynamics of the non-autonomous equation

$$
\begin{aligned}
& u_{t}=u_{x x}+u-\beta(t) u^{3}, x \in(0, \pi), t>s, \\
& u(0, t)=u(\pi, t)=0, t \geq s, \\
& u(x, s)=\phi(x), x \in[0, \pi] .
\end{aligned}
$$

If $\phi \in X_{1}^{ \pm}$, then $\phi(x)=\phi(\pi-x)$ and from the uniqueness of solution we have that $u(t, s, \phi)(x)=u(t, s, \phi)(\pi-x)$ for all $t \geq s$. Using comparison, it is easy to see that $X_{1}^{ \pm}$is invariant.

For $X_{2}^{ \pm}$we first note that if $\phi \in X_{2}^{ \pm}, \phi(x)=-\phi(\pi-x)$. It is clear that $\phi_{1,2}^{ \pm}=\left(\frac{\beta_{1}}{\beta_{2}}\right)^{\frac{1}{2}} \phi_{1,1}^{ \pm}$and that $\phi, \phi_{1,1}^{ \pm}$and $\phi_{1,2}^{ \pm}$vanish on $\frac{\pi}{2}$. Consider the solution $u(t, s, \phi)(x)$ of the initial boundary value problem

$$
\begin{aligned}
& u_{t}=u_{x x}+u-\beta(t) u^{3}, x \in(0, \pi), t>s, \\
& u(0, t)=u(\pi, t)=0, t \geq s, \\
& u(x, s)=\phi(x), x \in[0, \pi] .
\end{aligned}
$$

The lap-number property of that $u(t, s, \phi)$ ensures that it will have only one zero in $(0, \pi)$ (at least for $t>s$ sufficiently close to $s$ ). It follows from the fact that $\phi(x)=-\phi(\pi-x)$ and from the uniqueness of solutions that $u(t, s, \phi)(x)=$ $-u(t, s, \phi)(\pi-x)$ and from this we obtain that $u(t, s, \phi)\left(\frac{\pi}{2}\right)=0$ for all $t>s$. We can now use comparison in each of the intervals $\left(0, \frac{\pi}{2}\right)$ and $\left(\frac{\pi}{2}, 1\right)$ to say that $u(t, s, \phi)$ remains in $Y_{i}^{ \pm}$for $t>s$. 
Before proving the invariance of $X_{3}^{ \pm}$we consider the invariance of $X_{4}^{ \pm}$. The above reasoning ensures that any solution $u(t, s, \psi)$ starting at $\psi \in X_{4}^{ \pm}$must satisfy $u(t, s, \psi)\left(\frac{\pi}{2}\right)=0$ for all $t>s$. Restricting this solution to $\left(0, \frac{\pi}{2}\right)$ we have a solution of

$$
\begin{aligned}
& u_{t}=u_{x x}+u-\beta(t) u^{3}, x \in\left(0, \frac{\pi}{2}\right), t>s \\
& u(0, t)=u\left(\frac{\pi}{2}, t\right)=0, t \geq s \\
& u(x, s)=\psi(x), x \in\left[0, \frac{\pi}{2}\right]
\end{aligned}
$$

The reasoning used for $X_{2}^{ \pm}$shows that $u(t, s, \psi)\left(\frac{\pi}{4}\right)=0$ for all $t>s$ and the fact that $u(t, s, \psi)(x)=-u(t, s, \psi)(\pi-x)$ implies that $u(t, s, \psi)\left(\frac{3 \pi}{4}\right)=0$ for all $t>s$. Also $u(t, s, \psi)(x)=-u(t, s, \psi)\left(\frac{\pi}{2}-x\right)$ for $x \in\left(0, \frac{\pi}{2}\right)$ and $u(t, s, \psi)\left(\frac{\pi}{2}+x\right)=$ $-u(t, s, \psi)(\pi-x)$ for $x \in\left(\frac{\pi}{2}, \pi\right)$. This ensures that a solution starting in $X_{4}^{+}$stays in $Y_{4}^{ \pm}$. Now, restricting to each of the intervals and using comparison one can prove that $X_{4}^{ \pm}$is invariant.

The case $X_{3}^{ \pm}$is a little more elaborate. Consider

$$
W_{4}^{ \pm}=\left\{\phi \in H_{0}^{1}\left(0, \frac{4 \pi}{3}\right): \phi(x)=-\phi\left(\frac{4 \pi}{3}-x\right) \text { for } x \in\left(0, \frac{4 \pi}{3}\right), \phi_{\left.\right|_{[0, \pi]}} \in X_{3}^{ \pm}\right\} .
$$

It follows from the above argument that $W_{4}^{ \pm}$is positively invariant for

$$
\begin{aligned}
& u_{t}=u_{x x}+u-\beta(t) u^{3}, x \in\left(0, \frac{4 \pi}{3}\right), t>s, \\
& u(0, t)=u\left(\frac{4 \pi}{3}, t\right)=0, t \geq s, \\
& u(x, s)=\bar{\psi}(x), x \in\left[0, \frac{4 \pi}{3}\right],
\end{aligned}
$$

and hence the restriction of a solution of this equation to $[0, \pi]$ is a solution for (3.9). Since $X_{3}^{ \pm}$is the restriction of $W_{4}^{ \pm}$to $[0, \pi]$ we obtain the invariance of $X_{3}^{ \pm}$.

This reasoning can now be continued indefinitely and hence $X_{i}^{ \pm}$is invariant for each $1 \leq i \leq N$. Given this we can now easily prove the following result.

Theorem 8. Given $\lambda \in\left(\lambda_{N}, \lambda_{N+1}\right)$ there are at least $2 N+1$ non-autonomous equilibria for (3.1).

Proof. Define $X_{i}^{ \pm}$as before. Observe that $X_{i}^{ \pm}$are metric subspaces of $H_{0}^{1}(0, \pi)$ with the metric induced by $H_{0}^{1}(0, \pi)$, which is invariant. Hence, the solution operator of (3.1) restricted to $X_{i}^{ \pm}$has a pullback attractor, and therefore there is at least one global solution in $X_{i}^{ \pm}$and consequently a non-autonomous equilibrium in the sense of Definition 7 .

\section{Remark 9.}

(a) If one applies the results of 13 to each of the intervals with endpoints the consecutive zeroes of $\phi_{i, j}^{ \pm}$, this ensures that the pullback attractor of the semigroup restricted to $X_{i}^{ \pm}$is a unique bounded global solution. This does not characterize the pullback attractor in the whole space $H_{0}^{1}(0, \pi)$, but it says that the same sequence of bifurcations seen in the autonomous problem is also seen in the non-autonomous one. 
The full characterization of the pullback attractor in this very simple example remains a very interesting problem. Unfortunately we are only able to characterize the pullback attractor fully in some very specific cases (essentially, uniformly small non-autonomous perturbations). Not even in the case of a periodic function $\beta(\cdot)$ have we been able to provide such a full characterization at present.

(b) We are now able partially to justify the term 'non-autonomous equilibria'. Theorem 10 below shows that, if $\beta \in(0, \infty)$ and $\beta_{\varepsilon}(t) \stackrel{\epsilon \rightarrow 0}{\longrightarrow} \beta$ (uniformly for $t \in \mathbb{R}$ ), then, for $\epsilon$ suitably small, there is a unique hyperbolic global solution associated to (3.1) (with $\beta(t)=\beta_{\epsilon}(t)$ ) uniformly close to each of the equilibria $\phi_{\beta}^{ \pm}$. Observe that therefore, for $\epsilon$ small enough, the 'nonautonomous equilibria' defined above are uniformly close to each of the equilibria $\phi_{\beta}^{ \pm}$of (3.9) with $\beta(\cdot) \equiv \beta$, and so they must coincide with the hyperbolic global solutions which, as described in [2], are the natural candidates for 'equilibria' in the non-autonomous context.

(c) The reasoning used above to obtain the non-autonomous equilibria can be carried out for more general non-linearities (e.g. if instead of $u-\beta(t) u^{3}$ we consider a dissipative non-linearity $\mathbb{R}^{2} \ni(t, u) \mapsto f(t, u) \in \mathbb{R}$ which is odd in the second variable). In this case we can still consider the spaces $Z_{i}^{ \pm}$, but with the additional restrictions that near the zeros they are between two lines with slopes of the same sign going through the zero. While this will give the same bifurcation sequence for the non-autonomous equilibria, we may not be able to localize these, since we will not necessarily have 'bounding equilibria' $\phi_{i, j}^{ \pm}$from the autonomous problem.

\section{Structure of the Pullback attractor and Bifurcation Phenomena FOR SMALL NON-AUTONOMOUS PERTURBATIONS}

In this section we will consider what additional information we can obtain when $\beta(t)$ is a small non-autonomous perturbation of some constant value (which we take here to be 1). More precisely, we will fix $\lambda \in\left(\lambda_{N}, \lambda_{N+1}\right)$ for some $N$ and consider the family of problems

$$
\left\{\begin{array}{l}
u_{t}=u_{x x}+\lambda u-\beta_{\epsilon}(t) u^{3}, \quad x \in(0, \pi) \text { and } t>\tau, \\
u(0, t)=u(\pi, t)=0 \\
u(x, \tau)=\phi(x)
\end{array}\right.
$$

with $\epsilon \in[0,1], \beta_{\epsilon}(t) \in C^{1}(\mathbb{R}), \phi \in H_{0}^{1}(0, \pi)$, and

$$
\lim _{\epsilon \rightarrow 0} \sup _{t \in \mathbb{R}}\left|\beta_{\epsilon}(t)-1\right|=0 .
$$

As we have seen in the previous section, all the equilibria of (4.1) with $\epsilon=0$ are hyperbolic.

4.1. Structure of the pullback attractor. The following theorem is a consequence of the results in [2] and [4].

Theorem 10. There is an $\epsilon_{0}>0$ and a $\delta>0$ such that, for all $0 \leq \epsilon \leq \epsilon_{0}$ :

- There is a unique trajectory $\xi_{n, \epsilon}^{ \pm}: \mathbb{R} \rightarrow H_{0}^{1}(0, \pi)$ such that

$$
\left\|\xi_{n, \epsilon}^{ \pm}(t)-u_{n}^{ \pm}\right\|_{H_{0}^{1}}<\delta \quad \text { for all } t \in \mathbb{R}, \quad \text { for } 0 \leq n \leq N,
$$

with the convention that $u_{0}^{ \pm} \equiv 0$, 
- $\xi_{n, \epsilon}^{ \pm}(\cdot)$ is hyperbolic,

- and $\sup _{t \in \mathbb{R}}\left\|\xi_{n, \epsilon}^{ \pm}(t)-u_{n}^{ \pm}\right\|_{H_{0}^{1}} \rightarrow 0$ as $\epsilon \rightarrow 0$.

- Problem (4.1) has a pullback attractor $\left\{A_{\epsilon}(t): t \in \mathbb{R}\right\}$ and

$$
A_{\epsilon}(t)=\bigcup_{0 \leq n \leq N}\left(W^{u}\left(\xi_{n}^{+}\right)(t) \cup W^{u}\left(\xi_{n}^{-}\right)(t)\right) ;
$$

i.e. the pullback attractor is 'gradient-like'.

Let $\mathcal{S}_{\epsilon}=\left\{\xi_{n, \epsilon}^{ \pm}(\cdot): 0 \leq n \leq N\right\}$.

- For each bounded global solution $\xi_{\epsilon}: \mathbb{R} \rightarrow H_{0}^{1}(0, \pi)$ there are $\xi_{i}^{*}, \xi_{j}^{*} \in \mathcal{S}_{\epsilon}$ such that

$$
\lim _{t \rightarrow-\infty}\left\|\xi_{\epsilon}(t)-\xi_{i, \epsilon}^{*}(t)\right\|_{H_{0}^{1}}=0 \text { and } \lim _{t \rightarrow+\infty}\left\|\xi_{\epsilon}(t)-\xi_{j, \epsilon}^{*}(t)\right\|_{H_{0}^{1}}=0 .
$$

- $\mathcal{S}_{\epsilon}$ does not have any homoclinic structure (see [4]).

- The family of attractors $\left\{A_{\epsilon}(t): t \in \mathbb{R}\right\}$ is continuous; that is,

$$
\operatorname{dist}\left(A_{\epsilon}(t), A_{0}\right)+\operatorname{dist}\left(A_{0}, A_{\epsilon}(t)\right) \rightarrow 0 \quad \text { as } \quad \epsilon \rightarrow 0 .
$$

- There is a constant $\gamma>0$ such that for any bounded subset $B$ of $H_{0}^{1}(0, \pi)$,

$$
\operatorname{dist}\left(S_{\epsilon}(t, s) B, A_{\epsilon}(t)\right) \leq c(B) \mathrm{e}^{-\gamma(t-s)} .
$$

In any small non-autonomous perturbation of a gradient dynamical system, one can obtain a full description of the geometrical structure of the associated pullback attractor as above. The global attractor $\mathcal{A}$ of the autonomous equation (2.1) is what 11 defined as a 'regular attractor'; our results give a non-trivial example of what one could define as a 'regular pullback attractor'.

4.2. Bifurcations in the pullback attractor as $\lambda$ is varied. While the previous result gives a complete description of the pullback attractor for some fixed $\lambda$ and $\epsilon \leq \epsilon_{0}$, the value of $\epsilon_{0}$ depends on $\lambda$. In this section we show how the above result can be strengthened to provide a detailed description of the structure of the pullback attractor for a large range of $\lambda$; the essential (and unfortunate) feature is that we have to exclude a small interval either side of each of the 'bifurcation values' $\lambda_{n}$.

Proposition 11. For each $n \in \mathbb{N}$ and $\delta \in\left(0, \frac{3}{2}\right)$ there exists $\epsilon_{0}(n, \delta)$ such that

$$
A_{\lambda, \epsilon}(t)=\bigcup_{0 \leq i \leq n}\left(W^{u}\left(\xi_{n}^{+}\right)(t) \cup W^{u}\left(\xi_{n}^{-}\right)(t)\right),
$$

for all $\epsilon \leq \epsilon_{0}$ and all $\lambda \in\left[\lambda_{n}+\delta, \lambda_{n+1}-\delta\right]$.

Proof. Fix $n$ and $\delta$. Then, given $\tilde{\lambda} \in\left[\lambda_{n}+\delta, \lambda_{n+1}-\delta\right]$ there exist $\varepsilon:=\varepsilon(\tilde{\lambda})>0$ and $\tilde{\epsilon}_{0}(\tilde{\lambda})$ such that, for all $\epsilon \leq \epsilon_{0}$ and $\lambda \in \mathcal{O}_{\varepsilon}(\tilde{\lambda})$,

$$
A_{\lambda, \epsilon}(t)=\bigcup_{0 \leq i \leq n}\left(W^{u}\left(\xi_{n}^{+}\right)(t) \cup W^{u}\left(\xi_{n}^{-}\right)(t)\right) .
$$

As $J=\left[\lambda_{n}+\delta, \lambda_{n+1}-\delta\right] \times[0,1]$ is compact, we can take a finite recovering of $J$ by

$$
\mathcal{O}_{\varepsilon_{k}}\left(\lambda_{k}\right) \times\left[0, \tilde{\epsilon}_{k}\right], \quad k \in\{1, \ldots, N\} .
$$

Now, choose $\epsilon_{0}=\min _{1 \leq k \leq N} \tilde{\epsilon}_{k}$. Then, given $\lambda \in\left[\lambda_{n}+\delta, \lambda_{n+1}-\delta\right]$ there exists $k \in\{1, \ldots, N\}$ such that $\lambda \in \mathcal{O}_{\varepsilon_{k}}\left(\lambda_{k}\right)$. Thus, for any $\epsilon \leq \epsilon_{0}$ we get the result.

The following corollary is immediate. 
Corollary 12. Given $n_{0} \in \mathbb{N}$ and $\delta \in\left(0, \frac{3}{2}\right)$ there exists $\epsilon_{0}\left(n_{0}, \delta\right)$ such that for $\epsilon \leq \epsilon_{0}$,

$$
A_{\lambda, \epsilon}(t)=\bigcup_{0 \leq i \leq n}\left(W^{u}\left(\xi_{n}^{+}\right)(t) \cup W^{u}\left(\xi_{n}^{-}\right)(t)\right),
$$

for all $\lambda \in \bigcup_{n=0}^{n_{0}}\left[\lambda_{n}+\delta, \lambda_{n+1}-\delta\right]$.

\section{EXAmples With GRADIENT-LIKE PUllbaCK ATTRACTORS FOR FIXED VALUES OF $\lambda$}

In this section we consider three choices for $\beta(t)$ in which the attractor retains the gradient-like structure of the autonomous case. First we consider the case in which $\beta(t)$ has exponential dependence on $t$; here a rescaling of time leads to an autonomous Chafee-Infante equation and hence a complete description of the attractor. We then consider two further cases: first when $\beta(t)$ is slowly varying in time, and then in contrast when $\beta(t)$ is rapidly oscillating. Our approach in these latter cases is to fix $\lambda$ (with $\lambda \neq \lambda_{n}$ for all $n \in \mathbb{N}$ ) and then find suitable conditions (sufficiently slow variation and sufficiently fast oscillation) to ensure the gradient structure. While such time dependence is not 'close to' an autonomous function, we are able to reduce the problem to a small perturbation of an autonomous system.

5.1. Reduction to the autonomous case: $\beta(t)$ is exponential. With the particular choice

$$
\beta(t)=n_{\delta}(t):= \begin{cases}1 & t<0 \\ \mathrm{e}^{-\delta t} & t \geq 0\end{cases}
$$

for some $\delta>0$, the rescaled equation (3.4) becomes

$$
z_{t}=z_{x x}+ \begin{cases}\lambda z-z^{3} & t<0, \\ \left(\lambda-\frac{1}{2} \delta\right) z-z^{3} & t \geq 0 .\end{cases}
$$

The asymptotic behaviour of this equation as $t \rightarrow+\infty$ is the same as that of the autonomous equation

$$
z_{t}=z_{x x}+\left(\lambda-\frac{1}{2} \delta\right) z-z^{3}
$$

which is completely understood. In particular, if $\frac{1}{2} \delta>\lambda-\lambda_{1}$, then $z \equiv 0$ attracts all solutions as $t \rightarrow \infty$, while if $\frac{1}{2} \delta<\lambda-\lambda_{1}$, then (5.1) has a unique positive stationary solution $z_{\infty}(x)$ and all positive solutions satisfy $z(t) \rightarrow z_{\infty}$ in $X$ as $t \rightarrow \infty$.

Let $\mathcal{A}$ be the attractor of the problem

$$
z_{t}=z_{x x}+\lambda z-z^{3} .
$$

Then for $\lambda_{n+1}>\lambda>\lambda_{n}>\lambda-\frac{\delta}{2}>\lambda_{n-1}$, the pullback attractor $\{A(t): t \in \mathbb{R}\}$ of (3.1) is given by $A(t)=\mathcal{A}$ if $t<0$ and $A(t)=\mathrm{e}^{\delta t} T(t, 0) \mathcal{A}$ if $t \geq 0$.

If $\left\{0, z_{1}^{ \pm}, \ldots, z_{n-1}^{ \pm}\right\}$are the equilibria of (5.2), then all solutions $u(t)$ of (3.1) are asymptotic with respect to the relative error to $\left\{0, u_{1}^{ \pm}, \ldots, u_{n-1}^{ \pm}\right\}\left(u_{i}^{ \pm}=e^{\frac{\delta}{2} t} z_{i}^{ \pm}\right)$as $t \rightarrow \infty$; i.e., for each $u(t) \neq 0$ there exists $i \in\{0,1, \ldots, n\}$ such that, for + or - ,

$$
\lim _{t \rightarrow+\infty} \frac{\left\|u(t)-u_{i}^{ \pm}\right\|}{\left\|u_{i}^{ \pm}\right\|}=0
$$


On the other hand, if $\left\{0, u_{1}^{ \pm}, \ldots, u_{n}^{ \pm}\right\}$are the equilibria of (5.3) and $\left\{0, \xi_{1}^{ \pm}, \ldots\right.$, $\left.\xi_{n}^{ \pm}\right\}$are the global solutions of (3.1) that coincide with $\left\{0, u_{1}^{ \pm}, \ldots, u_{n}^{ \pm}\right\}$for $t<0$, then

$$
A(t)=\bigcup_{j=0}^{n} W^{u}\left(\xi_{j}^{ \pm}\right)(t) .
$$

Observe that some of the global solutions $\xi_{j}^{ \pm}$are not "hyperbolic" since they must be asymptotic as $t \rightarrow \infty$ to an equilibrium of (5.2) whose unstable manifold has a lower dimension than the equilibrium of (5.3) from which they come.

On the other hand, if, for $\delta>0$, we consider $\tilde{n}_{\delta}(t)$, where $\tilde{n}_{\delta}(t) \equiv 1$ if $t>0$ and $\tilde{n}_{\delta}(t)=\mathrm{e}^{-\delta t}$ for $t \leq 0$, then $u(x, t)$ is a solution of (3.1) if and only if $z(x, t)=$ $\tilde{n}_{\delta}(t)^{\frac{1}{2}} u(x, t)$ is a solution of

$$
\left\{\begin{array}{l}
z_{t}=z_{x x}+ \begin{cases}\left(\lambda-\frac{1}{2} \delta\right) z-z^{3} & t \leq 0 \\
\lambda z-z^{3} & t>0\end{cases} \\
z(0, t)=z(\pi, t)=0 \\
z(x, \tau)=\tilde{n}_{\delta}(\tau)^{\frac{1}{2}} \phi(x)
\end{array}\right.
$$

If $\mathcal{A}_{\delta}$ denotes the attractor of (2.1) with $\lambda$ replaced by $\lambda-\frac{\delta}{2}$, as above, the pullback attractor for (5.4) is given by $A(t)=\mathrm{e}^{\frac{\delta}{2} t} \mathcal{A}_{\delta}$ if $t<0$ and $T(t, 0) \mathcal{A}_{\delta}$ if $t \geq 0$.

In this second example, if we choose $\delta$ such that there is a positive integer $n_{0}$ such that $\left(n_{0}-1\right)^{2}<\lambda-\frac{\delta}{2}<n_{0}^{2}<\lambda<\left(n_{0}+1\right)^{2}$, the pullback attractor is strictly smaller than the forwards attractor. It is important to notice that some of the solutions in the forwards attractor may never be approximated by solutions in the pullback attractor for, due to the lap-number property of solutions of these one-dimensional parabolic problems (cf. Section 3.5), the solutions in the pullback attractor may have at most $n_{0}$ zeroes in $[0, \pi]$ whereas there will be solutions in the forwards attractor with $n_{0}+1$ zeroes.

In the next two sections we consider two particular forms of time dependence for a bounded $\beta(t)$ and recover a sequence of bifurcations which coincide with those described in Section 4, where $\beta(t)$ was a small non-autonomous perturbation of a constant. However, in the cases we consider, $\beta(t)$ is not close to an autonomous function.

5.2. Slowly varying non-autonomous terms. First we study the case in which $\beta(t)$ may vary significantly, but only slowly, in $t$.

Proposition 13. Take $\omega>0$ and let $\beta(t)=b(\omega t)$, where $b: \mathbb{R} \rightarrow\left[n_{0}, N_{0}\right]$ with $\sup _{t \in \mathbb{R}}\left|\frac{b^{\prime}(t)}{b(t)}\right|<\infty$. For any fixed $\lambda \in\left(\lambda_{n}, \lambda_{n+1}\right)$, if $\omega$ is sufficiently small, there exist $2 n+1$ hyperbolic global solutions $\left\{\xi_{0}, \xi_{1}^{ \pm}, \ldots, \xi_{n}^{ \pm}\right\}$such that

$$
A(t)=\bigcup_{j=0}^{n} W^{u}\left(\xi_{j}^{ \pm}\right)(t) \quad \text { for all } t \in \mathbb{R} .
$$

Proof. Setting $z(x, t)=\beta(t)^{\frac{1}{2}} u(t)$, we obtain the equation

$$
\left\{\begin{array}{l}
z_{t}=z_{x x}+\left(\lambda+\frac{\omega b^{\prime}(\omega t)}{b(\omega t)}\right) z-z^{3}, 0 \leq x \leq \pi \text { and } t>\tau, \\
z(0, t)=z(\pi, t)=0 \\
z(x, \tau)=b(\omega \tau)^{\frac{1}{2}} \phi(x) .
\end{array}\right.
$$


For $\lambda \in\left(\lambda_{n}, \lambda_{n+1}\right)$ the autonomous system

$$
\left\{\begin{array}{l}
z_{t}=z_{x x}+\lambda z-z^{3}, 0 \leq x \leq \pi \text { and } t>\tau, \\
z(0, t)=z(\pi, t)=0 \\
z(x, \tau)=\phi(x)
\end{array}\right.
$$

has $2 n+1$ hyperbolic equilibria $\left\{u_{0}, u_{1} \pm, \ldots, u_{n}^{ \pm}\right\}$and the semigroup associated to (5.6) is gradient. From Theorem 10, for suitably small $\omega$, equation (5.5) gives rise to an evolution process $S_{\omega}(t, s)$ which has $2 n+1$ hyperbolic global solutions $\left\{\xi_{0}, \xi_{1}^{ \pm}, \ldots, \xi_{n}^{ \pm}\right\}$and whose pullback attractor is gradient-like in the sense of [3]. Changing back to the original variables, the process $S(t, s)$ associated to (3.1) has $2 n+1$ hyperbolic global solutions

$$
\left\{\beta(t)^{-\frac{1}{2}} \xi_{0}, \beta(t)^{-\frac{1}{2}} \xi_{1}^{ \pm}, \ldots, \beta(t)^{-\frac{1}{2}} \xi_{n}^{ \pm}\right\}
$$

and a gradient-like attractor as in (4.3).

One could, of course, use similar arguments to those of Section 4.2 to obtain a result similar to Corollary 12 for this case.

5.3. Rapidly oscillating non-autonomous terms. Now consider the following case:

Proposition 14. Choose $b: \mathbb{R} \rightarrow\left[n_{0}, N_{0}\right]\left(0<n_{0}<N_{0}\right)$ such that

$$
\frac{b^{\prime}(t)}{b(t)}=h(t) \sin k t,
$$

where $0<h_{0} \leq h(t) \leq H_{0}$ for all $t \in \mathbb{R}$. For any fixed $\lambda \in\left(\lambda_{n}, \lambda_{n+1}\right)$, if $k$ is sufficiently large, then there exist $2 n+1$ hyperbolic global solutions $\left\{\xi_{0}, \xi_{1}^{ \pm}, \ldots, \xi_{n}^{ \pm}\right\}$ such that

$$
A(t)=\bigcup_{j=0}^{n} W^{u}\left(\xi_{j}^{ \pm}\right)(t) \text { for all } t \in \mathbb{R} .
$$

Proof. Note that $\int_{t_{1}}^{t_{2}} h(t) \sin k t \mathrm{~d} t \rightarrow 0$ as $k \rightarrow \infty$ (this is a consequence of the Riemann-Lebesgue Lemma). It follows from results on robustness of exponential dichotomies (see Theorem 7.6.11 in [8]) that given a hyperbolic equilibrium $u^{*}$ of (5.6), for suitably large $k$ the process associated to

$$
\left\{\begin{array}{l}
z_{t}=z_{x x}+(\lambda+h(t) \sin (k t)) z-3\left(u^{*}\right)^{2} z, 0 \leq x \leq \pi \text { and } t>\tau, \\
z(0, t)=z(\pi, t)=0 \\
z(x, \tau)=\phi(x)
\end{array}\right.
$$

also has an exponential dichotomy with constant, exponent, and projection close to those for the linearization of (5.6) around $u^{*}$, and hence there is a unique global solution near $u^{*}$, which is still hyperbolic.

It is also not difficult to see, from Theorem 3.9 in [4, that the process associated to

$$
\left\{\begin{array}{l}
z_{t}=z_{x x}+\left(\lambda+\frac{b^{\prime}(t)}{2 b(t)}\right) z-z^{3}, 0 \leq x \leq \pi \text { and } t>\tau, \\
z(0, t)=z(\pi, t)=0 \\
z(x, \tau)=\phi(x)
\end{array}\right.
$$

possesses a gradient-like pullback attractor. 
This follows from a result completely analogous to Theorem 10 that can be proved by simply proving that near a hyperbolic equilibrium for (5.9) with $b \equiv 1$ there is a unique hyperbolic global solution for (5.9) with $\frac{b^{\prime}(t)}{2 b(t)}=\sin (k t) h(t)$.

Note that, in this case, $\frac{b^{\prime}(t)}{b(t)}$ is no longer small (though bounded) and nevertheless the attractor remains gradient-like as in the case $b=1$.

\section{Conclusion}

We have applied results from a number of papers to study the structure of the pullback attractor for the non-autonomous model

$$
u_{t}=u_{x x}+\lambda u-\beta(t) u^{3} .
$$

Despite the fact that we have a full understanding of the autonomous version of this equation, the structure of the pullback attractor is still not clear in the general context $\beta_{1} \leq \beta(t) \leq \beta_{2}$. In addition to the desire for a 'full characterization' of the attractor, there are some more tractable and striking open questions:

- For $\lambda \in\left(\lambda_{1}, \lambda_{2}\right)$, is the pullback attractor in $X$ the union of those in the positive and negative cones (see Section 3.3)?

- How can one make the most profitable use of lap-number techniques in the non-autonomous case (cf. Section 3.5)?

- Can one say anything about the behaviour of the pullback attractor when $\beta(t) \simeq \beta$ (i.e. for a small non-autonomous perturbation) near the autonomous bifurcation values $\lambda=\lambda_{n}$ ?

We hope that this paper will highlight some of the interesting questions that are still open for very simple non-autonomous systems, in particular for the non-autonomous Chafee-Infante equation studied here, which we present as a canonical model worthy of further investigation.

\section{ACKNOWLEDGEMENT}

The authors want to express their sincere gratitude to the referee for the corrections, interesting comments and suggestions. We are especially thankful for the comments on Theorem 8 , which led to a better explanation of the invariance of the sets $X_{i}^{ \pm}$.

\section{REFERENCES}

[1] A. V. Babin and M. I. Vishik, Attractors in Evolutionary Equations, Studies in Mathematics and its Applications 25, North-Holland Publishing Co., Amsterdam, 1992. MR 1156492 (93d:58090)

[2] A. N. Carvalho and J. A. Langa, Non-autonomous perturbation of autonomous semilinear differential equations: Continuity of local stable and unstable manifolds, J. Differential Equations 233 (2007), 622-653. MR2292521 (2008f:37174)

[3] A. N. Carvalho, J. A. Langa, J. C. Robinson and A. Suárez, Characterization of nonautonomous attractors of a perturbed infinite-dimensional gradient system, J. Differential Equations 236 (2007), no. 2, 570-603. MR2322025 (2008e:37075)

[4] A. N. Carvalho and J. A. Langa, An extension of the concept of gradient semigroups which is stable under perturbation, J. Differential Equations 246 (2009), 2646-2668. MR2503016 (2010a:37159)

[5] N. Chafee and E. F. Infante, A bifurcation problem for a nonlinear partial differential equation of parabolic type. Applicable Anal. 4 (1974/75), 17-37. MR0440205 (55:13084) 
[6] V. V. Chepyzhov and M. I. Vishik, Attractors for Equations of Mathematical Physics, Providence: AMS Colloquium Publications, vol. 49, 2002. MR1868930 (2003f:37001c)

[7] J. Hale, Asymptotic Behavior of Dissipative Systems, Providence: Math. Surveys and Monographs, A.M.S., 1998. MR941371 (89g:58059)

[8] D. Henry, Geometric theory of semilinear parabolic equations, Lecture Notes in Mathematics, 840, Berlin: Springer, 1981. MR610244 (83j:35084)

[9] J. A. Langa, J. C. Robinson, A. Suárez and A. Vidal-López, Structural stability of gradientlike attractors under non-autonomous perturbations, J. Differential Equations 234 (2007), 607-625. MR2300669 (2008c:37125)

[10] J. A. Langa, J. C. Robinson and A. Suárez, Stability, instability, and bifurcation phenomena in non-autonomous differential equations, Nonlinearity 15 (2002), 887-903. MR:1901112 (2003a:37063)

[11] J. A. Langa, J. C. Robinson, A. Rodríguez-Bernal and A. Suárez, Permanence and asymptotically stable complete trajectories for non-autonomous Lotka-Volterra models with diffusion, SIAM J. Math. Anal. 40 (6) (2009), 2179-2216. MR 2481291(2010i:35151)

[12] J. A. Langa, J. C. Robinson, A. Suárez and A. Vidal-López, The stability of attractors for non-autonomous perturbations of gradient-like systems, J. Differential Equations 234 (2) (2007), 607-625. MR2300669 (2008c:37125)

[13] J. A. Langa, A. Rodríguez-Bernal and A. Suárez, On the long time behavior of nonautonomous Lotka-Volterra models with diffusion via the sub-supertrajectory method, J. Differential Equations 249 (2010), 414-445. MR.2644122

[14] J. A. Langa and A. Suárez, Pullback permanence for non-autonomous partial differential equations, Electronic J. Differential Equations 2002, No. 72. MR1921145 (2004a:35105)

[15] T. Ma and S. Wang, Attractor bifurcation theory and its applications to Rayleigh-Bénard convection, Commun. Pure Appl. Anal. 2 (4) (2003), 591-599. MR2019070 (2004i:37153)

[16] H. Matano, Nonincrease of the lap-number of a solution for a one-dimensional semilinear parabolic equation, J. Fac. Sci. Univ. Tokyo Sect. IA Math. 29 (1982), no. 2, 401-441. MR672070(84m:35060)

[17] J. C. Robinson, Infinite-Dimensional Dynamical Systems. Cambridge Texts in Applied Mathematics (2001). MR1881888(2003f:37001a)

[18] R. Temam, Infinite dimensional dynamical systems in mechanics and physics. New York: Springer, 1988. MR0953967 (89m:58056)

Instituto de Ciências Matemáticas e de Computaço, Universidade de São PauloCampus de São Carlos, Caixa Postal 668, 13560-970 São Carlos SP, Brazil

E-mail address: andcarva@icmc.usp.br

Departamento de Ecuaciones Diferenciales y Análisis Numérico, Universidad de Sevilla, Apdo. de Correos 1160, 41080 Sevilla, Spain

E-mail address: langa@us.es

Mathematical Institute, University of Warwick, Coventry, CV4 7AL, United KingDOM

E-mail address: j.c.robinson@warwick.ac.uk 